z together tens of millions of times per second.

㟧 The resultant data are measured in inverse

ن्ن femtobarns $\left(\mathrm{fb}^{-1}\right)$, a unit roughly equivalent to

o 100 trillion collisions. In the past month alone, the $\mathrm{LHC}$ recorded $1 \mathrm{fb}^{-1}$ worth of collisions. By the end of the year it aims have captured at least $15 \mathrm{fb}^{-1}$ (see 'Smashing!').

To gather these data, researchers are pushing the collider in two ways: by accelerating the particles to ever-greater energies and by increasing the number of collisions. Higher energies allow heavier particles to pop into being, but it is the number of collisions that will determine whether physicists have enough data to declare a discovery. In the weeks ahead, scientists will pack more protons inside the machine and focus the particles as tightly as possible onto the collision points at the centre of the LHC's two biggest detectors. Already, "we've done humongously better than we thought we could", says Mike Lamont, the head of accelerator operations at CERN.

Every time two tightly packed bunches of protons cross, they generate not one collision, but on average 27, Lamont says. But within a few weeks, that number is expected to rise into the mid-30s, peaking at around 40 collisions per crossing. The two main detectors at the LHC were designed to handle only around two dozen collisions at once. But they have managed to cope so far.

Each detector is made up of layers of smaller detectors that record the tracks of debris coming from their centre. When a collision occurs, computers above the machine decide whether

\section{SMASHING!}

As the LHC ramps up its proton collisions it will generate staggering quantities of data.

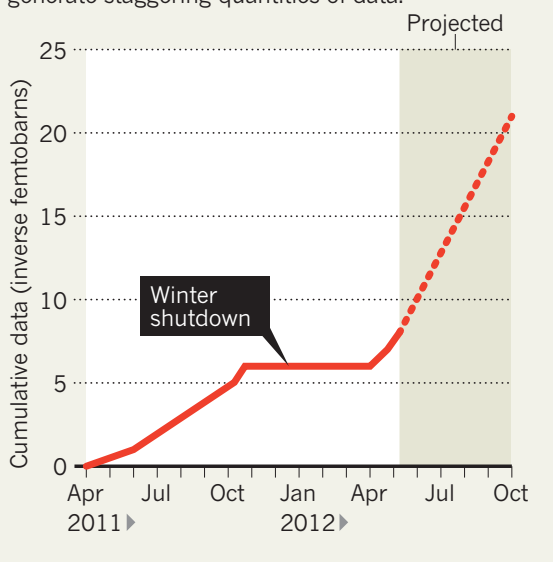

the data are interesting and, if so, reconstruct the collision from the tracks. But when dozens of collisions occur at once, the computers must disentangle them.

Last year, researchers working with the ATLAS detector formed a task force to tackle the pile-up problem, rewriting computer code so that the detector could cope with the extra collisions. Team member Andreas Salzburger says that the group has been working hard to weed out the 'ghost' particles that appear when the paths of several particles align, creating the illusion of a particle that is not actually there. Eliminating these ghosts as early as possible reduces the amount of computing power needed to crunch useful data, he says.

At the Compact Muon Solenoid (CMS), ATLAS's rival detector, physicists have trained their algorithms to triage data on the fly, analysing particle tracks in order of complexity. "Did you ever play the game 'pick-up sticks?," asks Joe Incandela, the spokesman for the CMS. "You pick up the easiest ones first, and it makes it simpler to deal with the other ones." The team is also working on ways to get rid of signals from 'loopers', low-energy particles that spiral along the detector's magnetic field lines, generating data that are irrelevant to the Higgs hunt.

Such tricks are likely to be less effective as the number of collisions rise. At the outer edges of the machine, the detector segments are larger and have coarser resolution, so it might not be possible to disentangle some of the tracks. That could reduce a detector's ability to pick up one signature of the Higgs: a decay to a pair of $\mathrm{W}$ bosons, which causes a cascade of particles that need to be caught by these outer segments.

For now, the mountains of extra data should offset what is lost to pile-up. Researchers expect to miss no more than $15 \%$ of events from the most likely Higgs decay pathway, which produces two $\gamma$-rays. And if ATLAS and the CMS can't handle the extra particles surging through the machine, Lamont says, the accelerator physicists are ready to dial it back. But "if they can take it, we will give it to them", he says.

\title{
A boost for quantum reality
}

\section{Theorists claim they can prove that wavefunctions are real states.}

\section{BY EUGENIE SAMUEL REICH}

$\mathrm{T}$ The philosophical status of the wavefunction - the entity that determines the probability of different outcomes of measurements on quantum-mechanical particles - would seem to be an unlikely subject for emotional debate. Yet online discussion of a paper claiming to show mathematically that the wavefunction is real has ranged from ardently star-struck to downright vitriolic since the article was first released as a preprint in November 2011.

The paper, thought by some to be one of the most important in quantum foundations in decades, was finally published last week in Nature Physics (M. F. Pusey, J. Barrett \& T. Rudolph Nature Phys. http://dx.doi. org/10.1038/nphys2309; 2012), enabling the authors, who had been concerned about violating the journal's embargo, to speak about it publicly for the first time. They say that the mathematics leaves no doubt that the wavefunction is not just a statistical tool, but rather, a real, objective state of a quantum system. "People have become emotionally attached to positions that they defend with vague arguments," says Jonathan Barrett, one of the authors and a physicist at Royal Holloway, University of London. "It's better to have a theorem."

The authors have some heavyweights in their corner: their view was once shared by Austrian physicist and quantum-mechanics pioneer Erwin Schrödinger, who proposed in his famous thought experiment that a quantum-mechanical cat could be dead and alive at the same time. But other physicists have favoured an opposing view, one held by Albert Einstein: that the wavefunction reflects the partial knowledge an experimenter has about a system. In this interpretation, the cat is either dead or alive, but the experimenter does not know which. This 'epistemic' interpretation, many physicists and philosophers argue, better explains the phenomenon of wavefunction collapse, in which a quantum state is fundamentally changed by measuring it.

Barrett and his colleagues are following the approach of physicist John Bell, who in 1964 proved that quantum mechanics has another counterintuitive implication: that measurements on one particle can influence the state of another, distant particle, faster than the speed of light should allow. Bell's was a 'nogo' theorem: its strategy was to show that theories that do not allow faster-than-light 
- influences cannot reproduce the predictions of quantum mechanics. Similarly, the theorem proposed by Barrett and his colleagues shows that theories that treat the wavefunction in terms of lack of knowledge of a system's physical state will also fail to reproduce those predictions. Given how well-confirmed quantum mechanics is, the theorem suggests that such epistemic theories are wrong. "I hope this will take its place alongside Bell's theorem," says Barrett.

\section{GROUNDED IN REALITY}

If the wavefunction simply reflects the experimenter's uncertainty, then different wavefunctions could represent the same underlying reality, says Terry Rudolph, an author on the paper and a physicist at Imperial College London. Rudolph gives the example of a die that can be prepared to give either even numbers, with a $1 / 3$ probability of getting 2,4 or 6 ; or prime numbers, with a $1 / 3$ probability of getting 2,3 or 5 . The real state 2 can be produced by either preparation method, so the same reality underlies two different probabilistic models. The authors show, however, that the same reality cannot underpin different quantum states.

Their theorem does, however, depend on a controversial assumption: that quantum systems have an objective underlying physical state. Christopher Fuchs, a physicist at the Perimeter Institute in Waterloo, Canada, who has been working to develop an epistemic interpretation of quantum mechanics, says that he has avoided the interpretations that the authors exclude. The wavefunction may represent the experimenter's ignorance about measurement outcomes, rather than the underlying physical reality, he says. The new theorem doesn't rule that out.

Still, Matt Leifer, a physicist at University College London who works on quantum information, says that the theorem tackles a big question in a simple and clean way. He also says that it could end up being as useful as Bell's theorem, which turned out to have applications in quantum information theory and cryptography. "Nobody has thought if it has a practical use, but I wouldn't be surprised if it did," he says.

Because it is incompatible with quantum mechanics, the theorem also raises a deeper question: could quantum mechanics be wrong? Everyone assumes that it reigns supreme, but there is always a possibility that it could be overturned. So Barrett is now working with experimentalists to check predictions that differ between the theory and the epistemic accounts it conflicts with. "We don't expect quantum mechanics would fail this test, but we should still do it," he says.

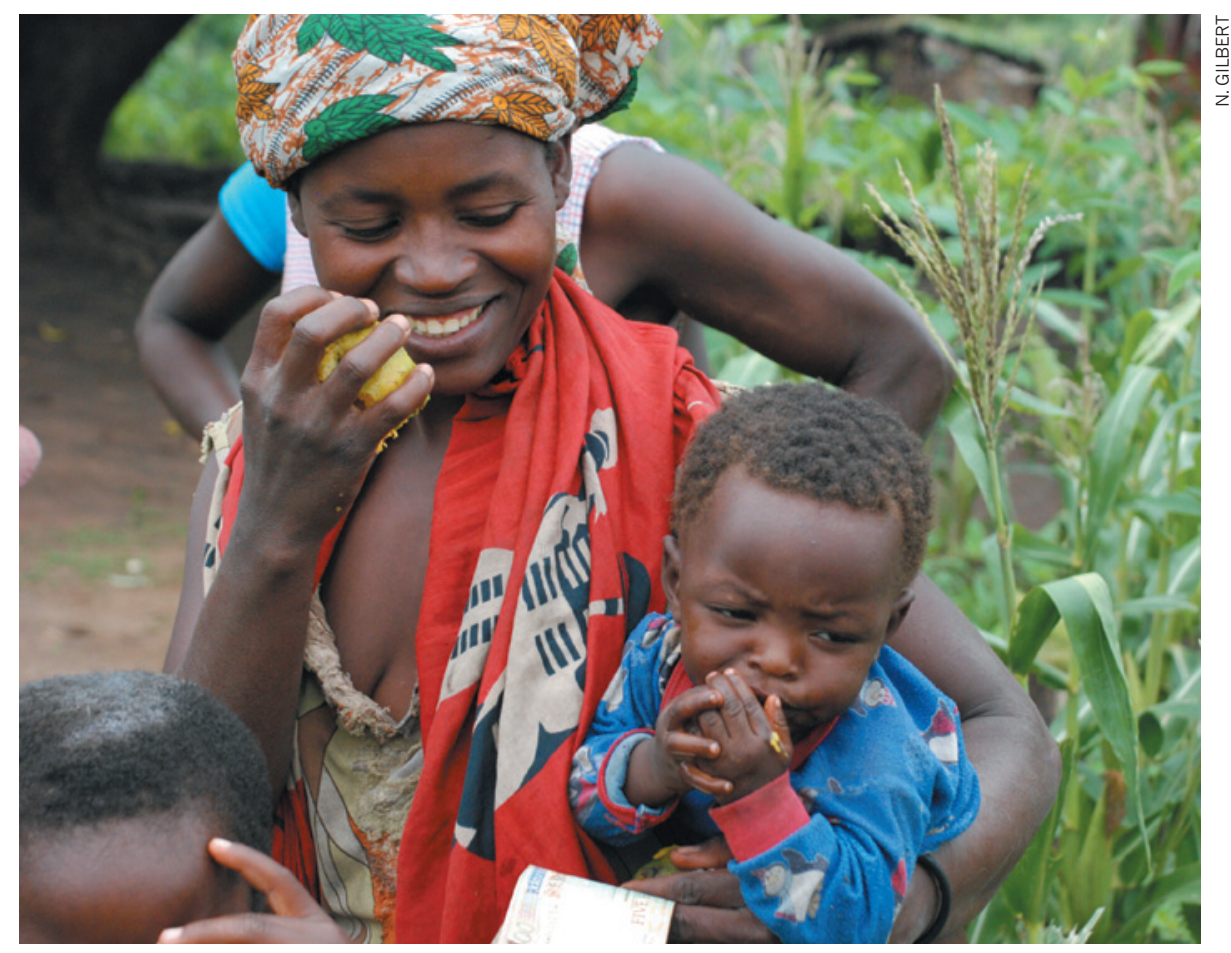

Children in Mwandama, Malawi, now have a better chance of living to the age of five.

GLOBAL HEALTH

\section{Development project touts health victory}

\author{
But critics question data and cost estimates from the \\ Millennium Villages Project.
}

\section{BY NATASHA GILBERT} or villagers in Mwandama, Malawi, visiting a health worker used to mean a daunting 40-kilometre round trip on foot. So the medical centre that was built in the area as part of the Millennium Villages Project (MVP) last year has improved their quality of life - and their health. Research published this week suggests that the MVP has significantly reduced infant mortality at sites across Africa.

But some researchers have questioned the methods used to quantify the benefits of the project, and demanded that the MVP release its underlying data. "The core of the problem is lack of transparency and careful, independent analysis," says Michael Clemens, a migration and development researcher at the Center for Global Development, an independent research institution in Washington DC.

\section{DNATURE.COM}

For more on science and development in Africa, see: nature.com/africa
$\mathrm{F}$
The MVP, which is spearheaded by Jeffrey Sachs, an economist at Columbia University in New York, aims to lift some of the poorest people in Africa out of poverty and improve their standard of living by boosting health and food security. It intends to help villages at 14 sites across Africa to reach the United Nations' eight Millennium Development Goals (MDGs) by 2015.

Sachs says that many aid projects see limited success because they focus on one area at a time. By contrast, the MVP tackles all the root causes of poverty at once. For example, it simultaneously provides free fertilizer and seeds, builds schools and gives business training to farmers. Funded by cash and in-kind contributions from governments, industry and aid donors, the project is growing in influence. The government of Cameroon is planning to start a similar scheme, for example, using funding from Japan and the UN to boost economic and employment opportunities for 50,000 villagers.

Research published in The Lancet (P. Pronyk 